\title{
On the Stability of Friedrichs' Scheme and the Modified Lax-Wendroff Scheme*
}

\author{
By Rémi Vaillancourt
}

Abstract. Necessary and sufficient stability criteria for Friedrichs' scheme and the modified Lax-Wendroff scheme with smooth coefficients are derived by means of Kreiss' Matrix Theorem and the first Stability Theorem of Lax and Nirenberg.

In this note we derive necessary and sufficient stability criteria for Friedrichs' scheme and the modified Lax-Wendroff scheme [8] for the hyperbolic system

$$
u_{t}=\sum_{i=1}^{n} a_{i}(x) u_{x_{j}}
$$

of first-order differential equations with variable coefficient matrices.

Friedrichs' scheme

$$
v(x, t+k)=S_{h} v(x, t)
$$

is given by the difference operator

$$
S_{h}=\frac{1}{2 n} \sum_{j}\left[T_{i}+T_{i}^{-1}\right]+\frac{1}{2} \lambda \sum_{i} a_{i}(x)\left[T_{i}-T_{i}^{-1}\right] \equiv C_{h}+\lambda A_{h},
$$

with $T_{i}$ representing the translation operator, by the amount $h$ in the $x_{i}$ direction. The symbol of $S_{h}$ is the matrix

$$
s(x, \xi)=\frac{1}{n}\left(\sum_{i} \cos \xi_{i}\right) I+i \lambda \sum_{i} a_{i}(x) \sin \xi_{i} \equiv c(\xi) I+i \lambda a(x, \sin \xi),
$$

where $\lambda=k / h, k$ being the time step and $h$ the space mesh size, $\xi=\left\{\xi_{1}, \cdots, \xi_{n}\right\}$, $\sin \xi=\left\{\sin \xi_{1}, \cdots, \sin \xi_{n}\right\}$, and $I$ is the identity matrix.

With the above notation, the modified Lax-Wendroff scheme [8] is given by

$$
v(x, t+k)=L_{k} v(x, t),
$$

where the operator

$$
L_{h} \equiv I+\lambda A_{h}\left[C_{h}+\frac{1}{2} \lambda A_{h}\right]
$$

Received May 26, 1969, revised December 1, 1969.

AMS 1969 subject classifications. Primary 6567; Secondary 3553, 3523.

Key words and phrases. Stability criteria, Friedrichs' scheme, modified Lax-Wendroff scheme, hyperbolic system, variable coefficients, Kreiss' Matrix Theorem, Lax-Nirenberg Stability Theorem, pseudo-difference schemes.

* The work presented in this paper is supported by the AEC Computing and Applied Mathematics Center, Courant Institute of Mathematical Sciences, New York University, under Contract AT(30-1)-1480 with the U. S. Atomic Energy Commission. Revised under Contract N00014-67-A0285-0010 with the Office of Naval Research. Reproduction in whole or in part is permitted for any purpose of the United States Government. 
has the symbol

$$
l(x, \xi)=I+i \lambda a(x, \sin \xi)\left[c(\xi) I+\frac{1}{2} i \lambda a(x, \sin \xi)\right] .
$$

A scheme is stable if $\|v(T)\| \leqq M(T)\|v(0)\|$, where $M$ is a function of $T$ but independent of $h$. The norm is ||$v(t) \|^{2}=\int|v(x, t)|^{2} d x$.

Kreiss' Matrix Theorem [1] states that a matrix $s(x, \xi)$ is stable if and only if there exists a positive-definite matrix $r(x, \xi)$ such that

$$
s^{*} r s \leqq r, \text { for all } x, \xi .
$$

If $s$ is normal, $s s^{*}=s^{*} s$, Kreiss' Theorem reduces to von Neumann's condition with $r=I$.

We shall prove our stability theorem under either one of the following assumptions.

(A) The matrices $a_{j}(x)$ are hermitian and twice continuously differentiable.

(B) The matrices $a_{j}(x)$ are infinitely differentiable, and constant for $|x|>R$; moreover the matrix

$$
a(x, \xi /|\xi|)=\sum_{i} a_{i}(x) \xi_{i} /|\xi|
$$

admits a positive-definite symmetrizer $r(x, \xi)$, which is infinitely differentiable in $x$, and $\xi \neq 0$.

If the system (1) is strictly hyperbolic it follows by a result of Kreiss that such an $r$ exists:

LEMMA [2]. A matrix a admits a positive-definite symmetrizer $r, r a=a^{*} r$, if and only if the eigenvalues of $a$ are real and its eigenvectors are linearly independent. In that case $r$ can be written in the form $r=b^{*} b$ where $d=b a b^{-1}$ is a diagonal matrix.

In fact, strict hyperbolicity of (1) means that the eigenvalues of $a(x, \xi /|\xi|)$ are real and distinct for all $x$, and $\xi \neq 0$; hence the homogenized eigenvectors, $w_{i}(x, \xi /|\xi|)$ i.e., the rows of $b(x, \xi)$, are linearly independent and depend smoothly on $x$, and $\xi \neq 0$. It follows that the symmetrizer $r$ is smooth in $x$, and $\xi \neq 0$.

Thus, under assumption (B) and by Kreiss' Lemma, the diagonal matrix

$$
d(x, \xi)=b(x, \xi) a(x, \xi /|\xi|) b^{-1}(x, \xi)
$$

is real. Moreover $d$ and $b$ are smooth in $x$ and $\xi \neq 0$, independent of $x$ for $|x|>R$, and homogeneous of degree zero in $\xi$; hence they are symbols of pseudo-difference schemes of Yamaguti and Nogi [9]. Such schemes $G_{h}$ with symbols $g(x, \xi)$ are defined by the formula

$$
\left(G_{h} u\right)(x)=\int e^{i x \cdot \xi} g\left(x, \lambda_{1}(h \xi)\right) a(\xi) d \xi,
$$

where we take $\lambda_{1}(\xi) \equiv \sin \xi$. For these schemes we have the following form of the Lax-Nirenberg Theorem [7]: If the matrix $g(x, \xi)$ is a hermitian and nonnegative symbol of the pseudo-difference scheme $G_{h}$, then

$$
\operatorname{Re}\left(G_{h} \Lambda_{h}^{2} u, u\right) \geqq-K h\|u\|^{2} .
$$

Here $\Lambda_{h}$ is the scheme with symbol $\left|\lambda_{1}(\xi)\right|$ and $K$ is a constant independent of $h$.

THEOREM. Under assumption (A) or (B), Friedrichs' scheme $S_{h}$ and the modified Lax-Wendroff scheme $L_{h}$ are stable if and only if their symbols $s$ and $l$ are stable. 
The following corollary gives sufficient conditions in terms of the absolute value of the largest eigenvalue of $a,|\sigma(a)|$.

COROLlaRY. Under assumption (A) or (B), $S_{h}$ is stable for those $\lambda$ satisfying

$$
\lambda|\sigma(a(x, \xi))| \leqq n^{-1 / 2},
$$

for all $x$ and $|\xi|=1$. Similarly $L_{h}$ is stable for $\lambda$ satisfying

$$
\lambda|\sigma(a(x, \xi))| \leqq 2 n^{-1 / 2} .
$$

Proof. We first prove the theorem and the corollary under assumption (A). In this case, the normal matrices $s$ and $l$ are stable if and only if

$$
|\sigma(s(x, \xi))| \leqq 1, \quad|\sigma(l(x, \xi))| \leqq 1 .
$$

Since each of these inequalities expresses simultaneously von Neumann's necessary condition and the Lax-Nirenberg sufficient condition [3], [4] for the stability of $S_{h}$ and $L_{h}$, the theorem follows. We deduce the corollary from the following inequalities

$$
\begin{aligned}
& p \equiv I-s^{*} s \geqq 0, \\
& q \equiv I-l^{*} l \geqq 0 .
\end{aligned}
$$

Using the identity

$$
\begin{aligned}
1-c^{2}(\xi) & =1-\left(\frac{1}{n} \sum_{i} \cos \xi_{i}\right)^{2} \\
& =\frac{1}{n} \sum_{i} \sin ^{2} \xi_{i}+\frac{1}{n^{2}} \sum_{i>k}\left(\cos \xi_{i}-\cos \xi_{k}\right)^{2},
\end{aligned}
$$

we obtain

$$
\begin{aligned}
p & \equiv p(\lambda)=I-c^{2}(\xi) I-\lambda^{2} a^{2}(x, \sin \xi) \\
& =\frac{1}{n}|\sin \xi|^{2} I+\frac{1}{n^{2}} \sum_{i>k}\left(\cos \xi_{i}-\cos \xi_{k}\right)^{2} I-\lambda^{2} a^{2}(x, \sin \xi) \\
& \geqq\left\{\frac{1}{n} I-\lambda^{2}\left[\sum_{i} a_{i}(x) \frac{\sin \xi_{i}}{|\sin \xi|}\right]^{2}\right\}|\sin \xi|^{2} \\
& \geqq\left\{\frac{1}{n}-\lambda^{2}\left|\sigma\left(a\left(x, \frac{\sin \xi}{|\sin \xi|}\right)\right)\right|^{2}\right\}|\sin \xi|^{2} I,
\end{aligned}
$$

whence (5) implies (8). Similarly, we find

$$
q \equiv q(\lambda)=\lambda^{2} a^{2}\left[I-c^{2} I-\frac{1}{4} \lambda^{2} a^{2}\right]=\lambda^{2} a^{2} p(\lambda / 2) .
$$

Thus (6) implies (9). Second, we consider assumption (B). By Kreiss' Lemma and Kreiss' Theorem the symbols $s$ and $l$ given by (2) and (3) respectively are stable if and only if

$$
\begin{aligned}
& p_{1} \equiv b_{1}^{*} b_{1}-s^{*} b_{1}^{*} b_{1} s \geqq 0, \\
& q_{1} \equiv b_{1}^{*} b_{1}-l^{*} b_{1}^{*} b_{1} l \geqq 0 .
\end{aligned}
$$

Here, $b_{1}=b(x, \sin \xi)$. With $d_{1}=d(x, \sin \xi),(10)$ and (4) give for (11) and (12)

$$
\begin{aligned}
p_{1} & \equiv p_{1}(\lambda) \\
& =b_{1}^{*}\left\{\frac{1}{n} I-\lambda^{2} d_{1}^{2}+\sum_{i>k}\left[|\sin \xi|^{-1}\left(\sin ^{2} \frac{\xi_{i}}{2}-\sin ^{2} \frac{\xi_{k}}{2}\right)\right]^{2} I\right\} b_{1}|\sin \xi|^{2} \geqq 0
\end{aligned}
$$


and

$$
q_{1} \equiv q_{1}(\lambda)=\lambda^{2} a_{1}^{*} p_{1}(\lambda / 2) a_{1}|\sin \xi|^{2} \geqq 0,
$$

with $a_{1}=a(x, \sin \xi /|\sin \xi|)$.

Since $a(x, \xi /|\xi|), b$ and $d$ are symbols of pseudo-difference schemes and the terms in the summation $\sum_{i>k}$ in (13) admit the calculus of these schemes, we can apply the Lax-Nirenberg Theorem [7] to $p_{1} \geqq 0$ in (13) to obtain

$$
\operatorname{Re}\left(P_{1, h} u, u\right) \geqq-K h\|u\|^{2} \text {. }
$$

with

$$
P_{1, h} u(x)=\int e^{i x \cdot \xi} p_{1}(x, h \xi) a(\xi) d \xi .
$$

It is shown in [9] that (15) implies the stability of $S_{h}$. Similarly (14) implies the stability of $L_{k}$. This proves the sufficiency of (11) and (12). The necessity of (11) and (12) is a consequence of Kreiss' Theorem and Kreiss' Lemma under assumption (B). The corollary follows also by the same theorem of Lax and Nirenberg [7].

This proves the theorem and the corollary.

If we require only Lipschitz continuity in assumption (A), the Lax-Wendroff Theorem [5] yields only strict inequality in (5) and (6) because it does not admit equality in (7). Whether the Lax-Nirenberg Theorem [3], [6] holds or not for Lipschitz coefficients is an open question. Assumption (B) on the $a_{j}(x)$ could be considerably weakened. The stability of $L_{h}$ for strictly hyperbolic systems (1) with constant coefficients was treated by Wendroff [8].

Department of Mathematics

The University of Chicago

Chicago, Illinois 60637

1. H. O. KREISs, "Uber die Stabilitätsdefinition für Differenzengleichungen, die partielle Differentialgleichungen approximieren," Nordisk Tidskr. Informations-Behandling, v. 2, 1962, pp. 153-181. MR 29 \#2292.

2. H. O. KREISs, "Uber sachgemässe Cauchyprobleme," Math. Scand., v. 13, 1963, pp. 109-128. MR 29 \#6177.

3. P. D. LAX \& L. Nirenberg, "On stability for difference schemes: A sharp form of Gårding's inequality," Comm. Pure Appl. Math., v. 19, 1966, pp. 473-492. MR 34 \#6352.

4. P. D. LAX \& L. Nirenberg, A Sharp Inequality for Pseudo-Differential and Difference Operators, Proc. Sympos. Pure Math., vol. 10, Amer. Math. Soc., Providence, R. I., 1967, pp. 213-217. MR 38 \#2424.

5. P. D. Lax \& B. Wendroff, "On the stability of difference schemes," Comm. Pure Appl. Math., v. 15, 1962, pp. 363-371. MR 27 \#4375.

6. R. VAillancourt, "A simple proof of Lax-Nirenberg theorems," Comm. Pure Appl. Math., v. 23, 1970, pp. 151-163.

7. R. VAILlanCOURT, "A strong form of Yamaguti and Nogi's stability theorem for Friedrichs' scheme," Publ. Res. Inst. Math. Sci. Ser. A, v. 5, 1969, pp. 113-117.

8. B. WENDROFF, "Well-posed problems and stable difference operators," SIAM J. Numer. Anal., v. 5, 1968, pp. 71-82. MR $36 \# 6159$.

9. M. YAMAGUTI \& T. NoGI, "An algebra of pseudo difference schemes and its applications," Publ. Res. Inst. Math. Sci. Ser. A, v. 3, 1967/68, pp. 151-166. MR 37 \#1762. 